\title{
NADPH-dependent thioredoxin reductase C plays a role in nonhost disease resistance against Pseudomonas syringae pathogens by regulating chloroplast-generated reactive oxygen species
}

Yasuhiro Ishiga, Takako Ishiga, Yoko Ikeda, Takakazu Matsuura, Kirankumar S Mysore

Chloroplasts are cytoplasmic organelles for photosynthesis in eukaryotic cells. In addition, recent studies have shown that chloroplasts have a critical role in plant innate immunity against invading pathogens. Hydrogen peroxide is a toxic by-product from photosynthesis, which also functions as a signaling compound in plant innate immunity. Therefore, it is important to regulate the level of hydrogen peroxide in response to pathogens.

Chloroplasts maintain components of the redox detoxification system including enzymes such as 2-Cys peroxiredoxins (2-Cys Prxs), and NADPH-dependent thioredoxin reductase $C$ (NTRC). However, the significance of 2-Cys Prxs and NTRC in the molecular basis of nonhost disease resistance is largely unknown. We evaluated the roles of Prxs and NTRC using knock-out mutants of Arabidopsis in response to nonhost Pseudomonas syringae pathogens. Plants lacking functional NTRC showed localized cell death (LCD) accompanied by the elevated accumulation of hydrogen peroxide in response to nonhost pathogens. Interestingly, the Arabidopsis ntrc mutant showed enhanced bacterial growth and disease susceptibility of nonhost pathogens. Furthermore, the expression profiles of the salicylic acid (SA) and jasmonic acid (JA) -mediated signaling pathways and phytohormone analyses including SA and JA revealed that the Arabidopsis ntrc mutant shows elevated JA-mediated signaling pathways in response to nonhost pathogen. These results suggest the critical role of NTRC in plant innate immunity against nonhost $P$. syringae pathogens. 


\section{NADPH-dependent thioredoxin reductase $\mathbf{C}$ plays a role in}

\section{2 nonhost disease resistance against Pseudomonas syringae}

\section{3 pathogens by regulating chloroplast-generated reactive}

\section{4 oxygen species}

5 Yasuhiro Ishiga ${ }^{1,2}$, Takako Ishiga ${ }^{2}$, Yoko Ikeda ${ }^{3}$, Takakazu Matsuura ${ }^{3}$ and Kirankumar S

6 Mysore $^{2}$

$7 \quad{ }^{1}$ Faculty of Life and Environmental Sciences, University of Tsukuba, 1-1-1 Tennodai, Tsukuba,

8 Ibaraki, Japan.

$9 \quad 2$ Plant Biology Division, The Samuel Roberts Noble Foundation, Ardmore, OK, USA.

$10{ }^{3}$ Institute of Plant Science and Resources, Okayama University, Kurashiki, Okayama, Japan.

12 Corresponding Author:

13 Yasuhiro Ishiga

14 Faculty of Life and Environmental Sciences, University of Tsukuba, 1-1-1 Tennodai, Tsukuba,

15 Ibaraki 305-8572, Japan.

16 Email address: ishiga.yasuhiro.km@u.tsukuba.ac.jp 


\section{Abstract}

19 Chloroplasts are cytoplasmic organelles for photosynthesis in eukaryotic cells. In addition, recent

20 studies have shown that chloroplasts have a critical role in plant innate immunity against

21 invading pathogens. Hydrogen peroxide is a toxic by-product from photosynthesis, which also

22 functions as a signaling compound in plant innate immunity. Therefore, it is important to

23 regulate the level of hydrogen peroxide in response to pathogens. Chloroplasts maintain

24 components of the redox detoxification system including enzymes such as 2-Cys peroxiredoxins

25 (2-Cys Prxs), and NADPH-dependent thioredoxin reductase C (NTRC). However, the

26 significance of 2-Cys Prxs and NTRC in the molecular basis of nonhost disease resistance is

27 largely unknown. We evaluated the roles of Prxs and NTRC using knock-out mutants of

28 Arabidopsis in response to nonhost Pseudomonas syringae pathogens. Plants lacking functional

29 NTRC showed localized cell death (LCD) accompanied by the elevated accumulation of

30 hydrogen peroxide in response to nonhost pathogens. Interestingly, the Arabidopsis ntrc mutant

31 showed enhanced bacterial growth and disease susceptibility of nonhost pathogens. Furthermore,

32 the expression profiles of the salicylic acid (SA) and jasmonic acid (JA) -mediated signaling

33 pathways and phytohormone analyses including SA and JA revealed that the Arabidopsis ntrc

34 mutant shows elevated JA-mediated signaling pathways in response to nonhost pathogen. These

35 results suggest the critical role of NTRC in plant innate immunity against nonhost $P$. syringae

36 pathogens.

\section{Introduction}


39 Plants are surrounded by a large number of microbes, including potential pathogens, in their

40 natural habitat. Thus, plants have evolved specific immune systems to defend themselves against

41 invading pathogens by developing a wide variety of constitutive and inducible defenses.

42 Constitutive defenses include preformed defense structures including cell walls, surface waxes,

43 the position of stomata, and preformed antimicrobial compounds. In addition to constitutive

44 defenses, plants have developed multiple layers of advanced surveillance systems against invading pathogens to activate a wide array of inducible defense responses including rapid oxidative burst, callose ( $\beta$-1,3-glucan) deposition in the cell wall, the induction of hormonemediated signaling pathways leading to the expression of defense-related genes, and the production of antimicrobial compounds (Hok et al., 2010). To date, these surveillance systems are known to include two layers of plant immune responses against invading pathogens. The first layer of plant immune responses is pathogen-associated molecular pattern (PAMP)-triggered immunity (PTI), which recognizes the conserved molecules from invading pathogens (microbes) using plant pattern-recognition receptors (PRRs) (Zipfel, 2008). The second layer of plant immune responses that exists to defend themselves from invading pathogens is effector-triggered immunity (ETI) based on the gene-for-gene interactions, which are governed by individual plant resistance $(R)$ genes and their corresponding avirulence (Avr) genes (Cui et al., 2014). ETI is often associated with the hypersensitive response (HR). The HR involves a wide array of responses including the activation of defense-associated genes, such as pathogenesis-related $(P R)$ genes, phytoalexin production, and the formation of rapid localized cell death (LCD) at the site of pathogen invasion (Coll et al., 2011). The HR-mediated LCD is the well-characterized form of plant programmed cell death (PCD) (Greenberg and Yao, 2004). Although the precise role of

61 death signals associated with the LCD is still unknown, the most investigated signal for LCD is 
62 reactive oxygen species (ROS) (Mur et al., 2008). Apoplastic ROS derived from plasma

63 membrane-bound NADPH oxidase is well known to regulate LCD (Mur et al., 2008). In addition

64 to apoplastic ROS, chloroplast-derived ROS have been shown to function in LCD (Ishiga et al.,

65 2009; Zurbriggen et al., 2009; Ishiga et al., 2012). Zurbriggen et al. reported the requirement of

66 chloroplast-generated ROS for LCD, based on tobacco plants over-expressing a chloroplast-

67 targeted cyanobacterial flavodoxin (Zurbriggen et al., 2009). Lim et al. demonstrated that loss-

68 of-function analysis of SlFTR-c (ferredoxin:thioredoxin reductase-c) resulted in spontaneous

69 necrosis development associated with elevated accumulation of ROS without pathogen

70 inoculation (Lim et al., 2010).

71 The chloroplast is the organelle which conducts photosynthesis to fix carbon in nature. In

72 addition, chloroplasts carry out a number of other functions, including fatty acid biosynthesis and

73 amino acid biosynthesis (Rolland et al., 2012). The biosynthesis of plant immunity-related

74 phytohormones including SA and JA occurs in the chloroplast (Wasternack and Hause, 2013;

75 Seyfferth and Tsuda, 2014). It was demonstrated that PTI signals are transmitted to the

76 chloroplast and activate $\mathrm{Ca}^{(2+)}$ signaling in the stroma through a calcium-sensing receptor

77 (Nomura et al., 2012). A recent study demonstrated that chloroplasts extend chloroplast

78 stromules to nuclei during plant immunity as well as reactive oxygen species (ROS) stress

79 (Brunkard et al., 2015; Caplan et al., 2015). Therefore, chloroplasts have a critical role in plant

80 immunity against pathogens. Furthermore, the chloroplast produces retrograde signals, such as

81 ROS, to regulate the nuclear gene expression in order to modulate chloroplast biogenesis,

82 maintain homeostasis, or optimize chloroplast performance under stress conditions, including

83 pathogen attacks (Chi et al., 2013; Chi et al., 2015). Low levels of ROS are known to function as

84 a retrograde signal, whereas excessive levels of ROS are known to cause oxidative damage 
85 resulting in LCD (Hossain et al., 2015). Therefore, plants have developed suitable mechanisms

86 to modulate the excessive production of ROS in the chloroplast. A chloroplast ROS

87 detoxification mechanism includes several antioxidant enzymes such as ascorbate peroxidase

88 (APX), glutathione peroxidase (GPX), and peroxiredoxin (Prx) (Gill and Tuteja, 2010). Prxs are

89 known to function together with NADPH dependent thioredoxin reductase (NTRC), which is a

90 central player in the chloroplast redox detoxification system in Arabidopsis plants (Kirchsteiger

91 et al., 2009; Bernal-Bayard et al., 2012; Bernal-Bayard et al., 2014; Puerto-Galán et al., 2015). It

92 has been shown that there are four chloroplast Prx isoforms in Arabidopsis, including PrxA,

93 PrxB, PrxQ, and PrxIIE (Tripathi et al., 2009).

94 We have previously shown that loss-of-function analysis of $\operatorname{Prx}$ and NTRC resulted in

95 accelerated Pseudomonas syringae pv. tomato DC3000 (Pto DC3000) disease associated cell

96 death with enhanced ROS accumulation in tomato and Arabidopsis (Ishiga et al., 2012). Our

97 previous studies also demonstrated that Pto DC3000 targets the chloroplast ROS homeostasis

98 and enhances ROS accumulation by suppressing the expression of genes encoding chloroplast

99 ROS detoxification enzymes including APX, Prx, and NTRC in Arabidopsis and tomato during

100 pathogenesis (Ishiga et al., 2009; Ishiga et al., 2012). This indicates that the NTRC/Prx

101 chloroplast ROS detoxification system functions as a negative regulator of disease-associated

102 cell death.

103 Nonhost resistance is known as the most common and durable form of plant disease resistance

104 against almost all microbes (including parasites and pathogens) in nature. Nonhost resistance is

105 believed to be regulated by many genes and to be involved in multiple layers, including

106 constitutive and inducible defense responses such as PTI and ETI (Mysore and Ryu, 2004; Niks

107 and Marcel, 2009; Fan and Doerner, 2012; Senthil-Kumar and Mysore, 2013). However, nonhost 
108 resistance is one of the least understood forms of resistance and is believed to be more durable

109 than host specific resistance in the field (Gill et al., 2015). The molecular basis of host specific

110 resistance has been well characterized. Unlike host resistance, a limited number of players

111 underlying nonhost resistance have been identified (Fan and Doerner, 2012; Senthil-Kumar and

112 Mysore, 2013). Therefore, an understanding of the molecular basis of nonhost resistance may

113 provide an avenue to engineer crop plants to confer durable resistance against a wide range of

114 pathogens.

115 Our previous results have shown that chloroplast ROS production plays a role in regulating

116 disease-associated cell death in tomato and Arabidopsis (Ishiga et al., 2009; Ishiga et al., 2012).

117 However, the precise role of chloroplast-derived ROS associated with LCD is still unclear. To

118 further understand the role of chloroplast-derived ROS in the interactions of plants and

119 pathogens, we investigated the functional analysis of the NTRC/Prx chloroplast ROS

120 detoxification system during nonhost resistance in Arabidopsis against Pseudomonas syringae,

121 and demonstrated that the ntrc mutant is compromised in nonhost resistance. Expression profiles

122 of SA- and JA-mediated signaling pathways and phytohormone analyses including SA and JA

123 identified a correlation between the JA-mediated signaling pathway and the enhanced

124 susceptibility of the $n \operatorname{trc}$ mutant against nonhost $P$. syringae. Thus, our results suggest the

125 importance of the NTRC/Prx chloroplast ROS detoxification system in plant immunity.

127 Materials and Methods

128 Plant materials and bacterial strains 
129 Arabidopsis thaliana ecotype Columbia (Col-0) was used as a wild-type plant in this study. $A$.

130 thaliana homozygous T-DNA insertion mutants prxA (At3g11630; CS875813), prxB

131 (At5g06290; SALK_017213), prxQ (At3g26060; SALK_070860), and prxIIE (At3g52960;

132 SALK_064512) were identified from the SALK Institute's collection (Ishiga et al., 2012). Seeds

133 of the Arabidopsis ntrc T-DNA mutant and its complemented line (Perez-Ruiz et al., 2006) were

134 kindly provided by Dr. Francisco Javier Cejudo (Instituto de Bioquímica Vegetal y Fotosíntesis,

135 Spain). Seeds of Arabidopsis plants were germinated and maintained on 1/2 Murashige and

136 Skoog (MS) medium (0.3\% phytagel) with Gamborg vitamins (Sigma-Aldrich, St. Louis, MO,

137 USA) and used for inoculation assays two weeks after germination at $25^{\circ} \mathrm{C}$ with a light intensity

138 of $200 \mu \mathrm{E} \mathrm{m}^{-2} \mathrm{sec}^{-1}$ and a $12 \mathrm{~h} \mathrm{light} / 12 \mathrm{~h}$ dark photoperiod.

139 Nonhost pathogens Pseudomonas syringae pv. tabaci (Pta) 6605 (Taguchi et al., 2001), P.

140 syringae pv. glycinea $(\mathrm{Pgl})$ race 4 (Staskawicz et al., 1984), and P. syringae pv. tomato (Pto) T1

141 (Almeida et al., 2009) were used to study nonhost resistance. P. syringae strains were grown at

$14228^{\circ} \mathrm{C}$ on mannitol-glutamate (MG) medium (Keane et al., 1970) for 36-48 h. Prior to inoculation,

143 bacteria were suspended in sterile distilled $\mathrm{H}_{2} \mathrm{O}$ and bacterial cell densities $\left(\mathrm{OD}_{600}\right)$ were

144 measured using a JASCO V-730 spectrophotometer (JASCO, Tokyo, Japan).

\section{Seedling flood-inoculation method}

146 Arabidopsis seedlings were inoculated by a method where MS agar plates were flooded with

147 bacterial cells as described previously (Ishiga et al., 2011). To perform uniform inoculation, 40

$148 \mathrm{ml}$ of bacterial suspension made in sterile distilled $\mathrm{H}_{2} \mathrm{O}$ containing $0.025 \%$ Silwet L-77 (OSI

149 Specialties Inc., Danbury, CT, USA.) was dispensed into the plate containing 2-week-old

150 Arabidopsis seedlings, and the plates were incubated for 2-3 min at room temperature. After the

151 bacterial suspension was removed by decantation, the plates containing inoculated plants were 
152 sealed with $3 \mathrm{M}$ Micropore $2.5 \mathrm{~cm}$ surgical tape (3M, St. Paul, MN, USA) and incubated at $24^{\circ} \mathrm{C}$

153 with a light intensity of $150-200 \mu \mathrm{E} \mathrm{m}^{-2} \mathrm{sec}^{-1}$ and a $12 \mathrm{~h}$ light/12 h dark photoperiod. Symptom

154 development was observed at 2 dpi. In each experiment, 8 plants were evaluated, and each

155 experiment was repeated at least three times.

156 To determine the bacterial growth in Arabidopsis leaves, we measured the internal bacterial

157 population at 2 dpi. For the determination of internal bacterial growth, inoculated seedlings were

158 collected by cutting the hypocotyls to separate the above agar parts (whole rosette) from the

159 Phytagel plate, and the total weight of the inoculated seedlings was measured. After

160 measurement of the seedlings' weight, the seedlings were surface-sterilized with $5 \% \mathrm{H}_{2} \mathrm{O}_{2}$ for 3

$161 \mathrm{~min}$. After washing three times with sterile distilled water, the plants were homogenized in sterile

162 distilled water, and the diluted samples were plated onto MG medium. Two days after plating of

163 the diluted samples, the bacterial colony forming units (CFU) were counted using proper diluted

164 samples. The CFU was normalized as CFU/mg using total weights of the inoculated seedlings.

165 Bacterial populations were evaluated in three independent experiments.

\section{Detection of cell death}

167 Cell death was estimated by measuring ion leakage from five plants treated with water (mock) or

168 inoculated with nonhost $P$. syringae pathogens and incubated at $24^{\circ} \mathrm{C}$ with a light intensity of

$169200 \mu \mathrm{E} \mathrm{m}^{-2} \mathrm{sec}^{-1}$ and a $12 \mathrm{~h}$ light/12 h dark photoperiod as described previously (Ishiga et al.,

170 2012). The inoculated plants were collected and then gently agitated in $30 \mathrm{ml}$ of distilled water

171 for $3 \mathrm{~h}$, and the leachates were measured using an ion conductivity meter (Omron, Kyoto, Japan).

172 The plants were then autoclaved for $20 \mathrm{~min}$ to kill the cells and release the total ions into the 
173 water. The values relative to the whole ion content after autoclaving were used to express the

174 percent ion leakage.

\section{Detection of hydrogen peroxide}

176 The generation of hydrogen peroxide was detected using 3,3'-diaminobenzidine (DAB) staining

177 as described previously (Ishiga et al., 2009; Ishiga et al., 2012; Rojas et al., 2012). To quantify

178 the accumulation of hydrogen peroxide over time, 10 leaves were collected at $24 \mathrm{~h}$ and $48 \mathrm{~h}$ after

179 inoculation with nonhost $P$. syringae pathogens, and then the leaves were placed in $1 \mathrm{mg} / \mathrm{ml}$

180 DAB-HCl (pH 3.8). After incubation for $6 \mathrm{~h}$ at room temperature, chlorophyll was removed with

$18195 \%$ ethanol and the leaves were mounted in 50\% glycerol. DAB staining in the leaves was

182 quantified using Image J software (version 1.49). The intensity of staining was expressed as a

183 percentage of coloration, where the intensity of coloration of the mock-inoculated wild-type Col-

1840 was set to $100 \%$.

\section{Quantification of phytohormones}

186 Approximately $100 \mathrm{mg}$ of fresh weight of two-week-old Arabidopsis plants grown on MS plates

187 were used for extraction. Extraction, purification, and quantification were performed as

188 described by Tsukahara et al. (Tsukahara et al., 2015). Quantification was performed by an

189 Agilent 1260-6410 Triple Quad LC/MS (Agilent Technologies Inc., Santa Clara, CA, USA)

190 equipped with a ZORBAX Eclipse XDB-C18 column (Agilent Technologies Inc.) using four

191 independent samples for each genotype.

\section{Real-time quantitative RT-PCR}

193 Total RNA extraction and real-time quantitative RT-PCR (qRT-PCR) were done as described 194 previously (Ishiga et al., 2013). Total RNA was extracted using RNAiso Plus (TaKaRa, Otsu, 
195 Shiga, Japan) according to the manufacturer's protocol. Two $\mu$ g of total RNA was treated with

196 gDNA Eraser (TaKaRa) to eliminate genomic DNA, and the DNase-treated RNA was reverse

197 transcribed using the PrimeScript ${ }^{\mathrm{TM}} \mathrm{RT}$ reagent Kit (TaKaRa). The cDNA (1:20) was then used

198 for qRT-PCR which was performed using the primers shown in Supplementary Table S1 with

199 SYBR $^{\circledR}$ Premix Ex Taq ${ }^{\text {TM }}$ II (TaKaRa) on a Thermal Cycler Dice ${ }^{\circledR}$ Real Time System (TaKaRa).

200 The Arabidopsis UBIQUITIN EXTENSION PROTEIN 1 (UBQ1) was used as an internal control

201 to normalize gene expression. The average CT values calculated using the 2 nd derivative

202 maximum method from triplicate samples were used to determine the fold expression relative to 203 the controls.

\section{Results}

An Arabidopsis ntrc mutant shows accelerated cell death in response to nonhost

\section{Pseudomonas syringae pathovars}

208 To investigate the role of chloroplast ROS homeostasis during nonhost resistance, we analyzed

209 four individual T-DNA insertional mutants deficient in chloroplast-localized peroxiredoxins

210 (PrxA, $\operatorname{PrxB}, \operatorname{Pr} x Q$, and $\operatorname{Pr} x I I E)$ and a T-DNA insertion mutant of $N T R C$, an electron donor for

211 Prxs in the Trx system (Tripathi et al., 2009; Cejudo et al., 2012). Two-week-old Arabidopsis

212 plants including Col-0 (wild-type), and the prx and ntrc mutants grown on MS agar were flood-

213 inoculated (Ishiga et al., 2011) with the non-host pathogens Pta 6605 (Taguchi et al., 2001), Pgl

214 race 4 (Staskawicz et al., 1984), and Pto T1 (Almeida et al., 2009). Nonhost bacterial pathogens

215 in Nicotiana benthamiana induce two categories of nonhost resistance responses: Type I, which

216 does not result in visible cell death; and Type II, which includes LCD in response to nonhost 
217 pathogens (Mysore and Ryu, 2004; Oh et al., 2006). We therefore first monitored for the Type I

218 and Type II response to nonhost pathogens in wild-type plants. Pta 6605 induced cell death

219 which was associated with a high percentage of ion leakage in the wild-type Col-0 (Figs. 1A and

220 1B). On the other hand, wild-type Col-0 plants inoculated with nonhost pathogens $P g l$ race 4 and

221 Pto T1 showed no obvious symptoms or ion leakage, and remained healthy (Figs. 1A and 1B).

222 These results indicate that Pta 6605 involves the Type I nonhost resistance, whereas $P g l$ race 4

223 and Pto T1 involve the Type II nonhost resistance in Arabidopsis. No significant difference was

224 observed in symptom development and ion leakage between the wild-type Col-0 and the prxa, 225 prxb, prxq, and prxIIe mutants in response to Pta 6605, Pgl race 4, and Pto T1 (Figs. 1A and 226 1B).

227 Interestingly, the knock-out mutant of ntrc showed accelerated cell death in response to Pta 6605

228 when compared to the wild-type Col-0 (Fig. 1A). Surprisingly, the type II nonhost pathogens $P g l$

229 race 4 and Pto T1 also induced cell death on $n t r c$ plants, unlike the wild-type Col-0 and the prx

230 mutants (Fig. 1A). Consistent with the visible phenotypes, a higher percentage of ion leakage

231 was observed in $n t r c$ mutant plants inoculated with non-host pathogens; however, Pta 6605

232 induced slightly stronger cell death in the ntrc mutant than the other nonhost pathogens tested

233 (Fig. 1B).

234 An Arabidopsis ntrc mutant shows enhanced susceptibility to nonhost Pseudomonas

235 syringae pathovars

236 To test if the severe disease symptoms are associated with increased bacterial multiplication in

237 the $n t r c$ mutant, the bacterial populations were monitored 2 days after inoculation with the 238 nonhost pathogens Pta 6605, Pgl race 4, and Pto T1. The bacterial populations of Pta 6605, Pgl

239 race 4, and Pto $\mathrm{T} 1$ in the $n t r c$ mutant were higher than those in the wild-type Col-0 and the prx 
240 mutants (Figs. 1C, 1D, and 1E). Interestingly, the $n t r c$ mutant inoculated with $P g l$ race 4 showed

241 significantly increased bacterial populations compared with the other nonhost pathogens (Figs.

$2421 \mathrm{C}, 1 \mathrm{D}$, and 1E). Furthermore, the complement line for the ntrc mutant showed almost identical

243 responses with the wild-type Col-0 against nonhost $P$. syringae pathogens (Supplementary Figs.

$2441 \mathrm{~A}, 1 \mathrm{~B}$, and $1 \mathrm{C})$. These results suggest that NTRC plays a role in the nonhost resistance response

245 in Arabidopsis.

246 An Arabidopsis ntrc mutant shows accelerated ROS accumulation in response to nonhost

247 Pseudomonas syringae pathovars

248 To investigate if nonhost pathogen-induced cell death is associated with accelerated ROS

249 accumulation, DAB staining was carried out in the wild-type Col-0, and the prx and ntrc mutants

250 after flood-inoculation with Pta 6605, $P g l$ race 4, and Pto T1. The accumulation of hydrogen

251 peroxide represented by the deposition of brown color was detected in the wild-type Col- 0 , and

252 the prx and ntrc mutants in response to nonhost pathogens. However, slightly elevated ROS

253 production was detected in the $n t r c$ mutants at $24 \mathrm{~h}$ after inoculation with Pta 6605 when

254 compared to $P g l$ race 4 and Pto T1 (Supplementary Fig. S2). As expected, a higher level of ROS

255 accumulation was observed in the ntrc mutant compared with those in the wild-type Col- 0 and

256 the prx mutants at $48 \mathrm{~h}$ after inoculation with nonhost pathogens (Figs. 2A and 2B).

257 Interestingly, higher ROS production (stronger DAB staining) was detected in the ntrc mutants at

$25848 \mathrm{~h}$ after inoculation with Pta 6605 and Pgl race 4 than with Pto T1 (Figs. 2A and 2B).

259 Furthermore, the complemented line for the ntrc mutant showed the same level of ROS

260 accumulation as the wild-type Col-0 in response to nonhost $P$. syringae pathogens

261 (Supplementary Figs. S1D and S1E). These results suggest that the severe cell death induced by

262 nonhost pathogens is associated with the accelerated ROS accumulation in the $n$ trc mutant. 
263 We next investigated the expression profiles of marker genes for chloroplast ROS accumulation,

264 including STROMAL ASCORBATE PEROXIDASE (sAPX), THYLAKOIDAL ASCORBATE

265 PEROXIDASE (tAPX), GLUTATHIONE PEROXIDASE 1 (GPX1), and GLUTATHIONE

266 PEROXIDASE 7 (GPX7) in response to inoculation with Pta 6605. Two-week-old Arabidopsis

267 plants, including the wild-type Col-0, the ntrc mutant, and the complemented line (35S-

268 NTRC/ntrc) were grown on MS plates, and were inoculated with Pta 6605, and then total RNA

269 was isolated from samples fixed at 3, 6, 12, and $24 \mathrm{~h}$ after inoculation. There was no significant

270 difference among the wild-type Col-0, the ntrc mutant, and the complemented line with respect

271 to the expression profiles of $s A P X, t A P X$, and $G P X 1$ (Supplementary Fig. S3A, S3B, and S3C).

272 The expression of GPX7 was only induced in the ntrc mutant when compared with the wild-type

273 Col-0 and complemented line (Supplementary Figs. S3D).

274

275

276

277

278

279

280

281

282

283

284

285

\section{An Arabidopsis ntrc mutant shows elevated JA-mediated signaling pathways in response to}

\section{Pta 6605}

The phytohormone-mediated signaling pathways leading to defense responses against invading pathogens have been shown to have a critical role in plant immunity (Robert-Seilaniantz et al., 2011; Pieterse et al., 2012). In general, the SA-mediated signaling pathway is implicated in the regulation of defense responses against biotrophic and hemibiotrophic pathogens, while the JA pathway is associated with defense responses against necrotrophic pathogens (Robert-Seilaniantz et al., 2011; Pieterse et al., 2012). Since several studies have demonstrated that phytohormonemediated signaling pathways play a role in nonhost resistance against fungal and bacterial pathogens (Loehrer et al., 2008; Ishiga et al., 2011; Lee et al., 2013), we next assessed the activities of the SA- and JA-mediated signaling pathways by investigating the accumulation of multiple phytohormones such as SA, JA, and JA-Ile, and the expression profiles of the SA- and 
286 JA-responsive genes in the wild-type Col-0, the $n \operatorname{trc}$ mutant, and the complement line (35S287 NTRC/ntrc).

288 Two-week-old Arabidopsis plants, including the wild-type Col-0, the ntrc mutant, and the 289 complement line (35S-NTRC/ntrc) were grown on MS plates, and were treated with water as a 290 control (mock), or inoculated with Pta 6605, and then the samples which were fixed at $24 \mathrm{~h}$ after 291 treatment or inoculation were utilized for the quantification of multiple phytohormones by LC292 ESI-MS/MS analysis. Interestingly, a higher level of JA and JA-Ile accumulation was observed 293 in the ntrc mutant plants in response to the inoculation with Pta 6605 compared with the wild294 type Col-0 and complement line (Figs. 3B and 3C), whereas no significant differences were 295 observed in the accumulation of SA between the wild-type Col-0 and the ntrc mutant (Fig. 3A), 296 indicating that NTRC may negatively regulate the JA biosynthesis pathway.

297 We next investigated the expression profiles of marker genes for the SA- and JA-signaling 298 pathways, including ISOCHORISMATE SYNTHASE 1 (ICS1), PATHOGENESIS-RELATED PROTEIN 1 (PR1), LIPOXYGENASE 2 (LOX2), 12-OXOPHYTODIENOIC ACID REDUCTASE 300 3 (OPR3), MYC2, GLUTAREDOXIN PROTEIN 480 (GRX480), NAC DOMAIN CONTAINING PROTEIN 19 (ANAC019), and JASMONATE-ZIM-DOMAIN PROTEIN 3 (JAZ3) in response to

302 inoculation with Pta 6605. Two-week-old Arabidopsis plants, including the wild-type Col-0, the 303 ntrc mutant, and the complement line (35S-NTRC/ntrc) were grown on MS plates, and were

304 inoculated with Pta 6605, and then total RNA was isolated from samples fixed at 3, 6, 12, and 24 $305 \mathrm{~h}$ after inoculation. The expression of SA-mediated signaling pathway genes including ICS1 and 306 PRI was induced by inoculation with Pta 6605 among the wild-type Col-0, the $n t r c$ mutant, and 307 the complement line (Figs. 4A and 4B). However, lower levels of the ICS1 and PRI transcripts 308 were observed in the ntrc mutant compared with the wild-type Col-0 and the complement line 
309 (Figs. 4A and 4B). In addition, the expression of JA biosynthesis genes including LOX2 and

310 OPR3, was induced in the ntrc mutant in response to inoculation with Pta 6605, but not in the

311 wild-type Col-0 and the complement line (Figs. 4C and 4D). Interestingly, the expression of key

312 regulators in the complex cross-talk between the SA- and JA-mediated signaling pathways

313 including $M Y C 2$, GRX480, ANAC19, and JAZ3 was significantly up-regulated in the ntrc mutant

314 in response to inoculation with Pta 6605. Taken together, these results indicate that the ntrc

315 mutant shows elevated JA-mediated signaling pathways in response to nonhost pathogens.

316

\section{Discussion}

318 In this study, we conducted the functional analysis of Prx and NTRC, which function as central

319 players of the chloroplast redox detoxification system in the nonhost disease resistance of

320 Arabidopsis against bacterial pathogens, and our results demonstrate the relevance of NTRC in

321 nonhost disease resistance against bacterial pathogens. Nonhost disease resistance can be divided

322 into Type I and Type II nonhost resistance based on the presence or absence of visual symptoms.

323 Type I nonhost resistance does not show any visible symptoms and involves PTI, whereas Type

324 II nonhost resistance is associated with visual symptoms because of the HR and involves ETI

325 (Mysore and Ryu, 2004; Niks and Marcel, 2009; Fan and Doerner, 2012; Senthil-Kumar and

326 Mysore, 2013). There is overlap between ETI and PTI regarding the signal transduction

327 pathways. In general, ETI is known as a more intense response that is often associated with the

328 HR. The HR involves a wide array of responses including the activation of defense-associated

329 genes, such as pathogenesis-related (PR) genes, phytoalexin production, and the formation of

330 rapid LCD at the site of pathogen invasion to restrict the growth of invading biotrophic and

331 hemibiotrophic pathogens. Both ETI and PTI are known to induce common signal transduction 
332 pathways including the oxidative burst and the activation of mitogen-activated protein kinase

333 (MAPK) pathways leading to the expression of defense-associated genes (Zipfel and Felix,

334 2005), indicating that Type I and Type II nonhost resistance involves these signal transduction

335 pathways. In this study, we investigated both Type I and Type II nonhost resistances in the ntrc

336 and prx mutants against nonhost bacterial pathogens. The results presented in Fig. 1

337 demonstrated that the ntrc mutant showed enhanced LCD not only to Type II nonhost pathogen

338 (Pta 6605), but also to Type I nonhost pathogens including Pto T1 and $P g l$ race 4 (Figs. 1 A and

339 1B). Interestingly, the LCD observed in the ntrc mutant was accompanied by the elevated

340 accumulation of chloroplast-derived ROS (Figs. 2A and 2B), suggesting the correlation between

341 LCD and chloroplast-derived ROS. ROS have been long recognized to orchestrate LCD, and the

342 plasma membrane-bound NADPH oxidase has been considered as the main source of ROS in

343 response to invading pathogens during ETI. However, there is increasing evidence for the role of

344 chloroplast-derived ROS in LCD (Liu et al., 2007; Zurbriggen et al., 2009). In support of our

345 results, a recent study demonstrated the requirement of chloroplast-derived ROS for the progress

346 of LCD using tobacco plants expressing a chloroplast-targeted cyanobacterial flavodoxin, which

347 functions as a general antioxidant to restrict the formation of ROS in the chloroplasts

348 (Zurbriggen et al., 2009). Moreover, they also indicated that chloroplast-derived ROS had no

349 significant effect on the regulation of genes related to defense and photosynthesis. Consistent

350 with these reports, we revealed that the ntrc mutant did not show any activation of SA-related

351 genes, such as ICSI and $P R-1$ accompanied by the accumulation of SA in response to nonhost

352 pathogens (Figs. 3A, 4A, and 4B). Taken together, these results suggest that chloroplast-derived

353 ROS have an impact on the progress of LCD during the HR, but not on the activation of defense-

354 associated genes. Furthermore, it is likely that this hypothesis could elucidate the phenomena 
355 that the ROS marker genes including $S A P X, t A P X$, and GPXI were not activated in the ntrc

356 mutant during nonhost resistance.

357 In addition, a chloroplast redox homeostasis based on the ferredoxin:thioredoxin system was also

358 shown to regulate LCD in tomato (Lim et al., 2010). Interestingly, unlike the phenotypes shown

359 in the ntrc mutant in this study, Lim et al. (2010) showed that silencing of the tomato

360 ferredoxin:thioredoxin reductase (SlFTR-c) resulted in spontaneous LCD development even

361 without the pathogen inoculation. They also demonstrated that SlFTR-c-silenced plants showed

362 enhanced resistance to Pto DC3000 by activating the expression of $P R$-genes (Lim et al., 2010).

363 FTR reduces the thioredoxins (TRXs) with electrons derived from ferredoxin, and functions in

364 chloroplast redox homeostasis by regulating chloroplast enzymes. Therefore, it is likely that the

365 silencing of SlFTR-c may affect the chloroplast redox homeostasis. Moreover, Tada et al. (2008)

366 reported that redox balance change controlled the conformation of NPR1, a master regulator of

367 the SA-mediated signaling pathway leading to the expression of defense-associated genes (Tada

368 et al., 2008). They also demonstrated that SA-induced conformation changes in NPR1 were

369 regulated by TRXs (Tada et al., 2008). Thus, it is possible that the chloroplast redox change

370 because of the absence of FTR may affect the TRXs-dependent conformation change of NPR1,

371 resulting in the activation of $P R$-genes. In contrast with these results, we showed no obvious

372 activation of the SA-mediated signaling pathways in the ntrc mutant. NTRC uses NADPH to

373 detoxify the excessive production of chloroplast-derived ROS coupled with Prx (Perez-Ruiz et

374 al., 2006; Kirchsteiger et al., 2009; Bernal-Bayard et al., 2012; Bernal-Bayard et al., 2014;

375 Puerto-Galán et al., 2015). Together, these results suggest that although both the FTR and NTRC

376 systems play an important role in the chloroplast redox regulation, the precise functions for these

377 systems may diverge based on the target molecules. 
378 We showed that the loss-of-function of NTRC resulted in excessive ROS accumulation when

379 compared to the wild-type in response to nonhost bacterial pathogens (Fig. 2), causing enhanced

380 LCD. Furthermore, the ntrc mutant showed elevated growth of nonhost bacterial pathogens (Fig.

381 1). Therefore, one could argue how enhanced LCD can contribute to bacterial growth in the ntrc

382 mutant. One possibility that should be considered is that $P$. syringae pathovars could get

383 nutrients from the tissues showing LCD during the necrotrophic growth phase. P. syringae is a

384 hemibiotrophic bacterial pathogen that shows no symptoms during the biotrophic growth phase

385 including epiphytic colonization on the leaf surface and early apoplastic colonization. However,

386 once the bacterial population density grows to a particular quorum-sensing (QS) threshold, QS

387 molecules trigger the expression of a large number of $P$. syringae genes, which regulate the

388 formation of disease-associated cell death, resulting in a switch to the necrotrophic growth phase

389 (Newton et al., 2010). Although it has been considered that LCD is more effective in plant

390 defense systems against invading obligate biotrophic pathogens, LCD may enable necrotrophic

391 pathogens to multiply by providing nutrients from dead tissues (Mur et al., 2008; Coll et al.,

392 2011). It has been reported that some necrotrophic pathogens could promote the ROS

393 production. In addition, several $P$. syringae pathovars are known to produce nonhost-specific

394 phytotoxins to promote lesions associated with symptoms. For example, $P g l$ and Pta produce the

395 chlorosis-inducing phytotoxins coronatine (COR) and tabtoxin, respectively, for their

396 pathogenicity (Bender et al., 1999). Interestingly, in our previous study (Ishiga et al., 2009) and

397 an ultrastructural study by Palmer and Bender (1995) revealed that COR targets the chloroplast

398 for pathogenicity (Palmer and Bender, 1995; Ishiga et al., 2009). Palmer and Bender also

399 demonstrated that the apoplastic extracts from tomato leaves treated with COR could enhance

400 the multiplication of Pto, indicating that the leakage of nutrients from COR-treated tissues could 
401 be sufficient for Pto growth (Palmer and Bender, 1995). Furthermore, it has been well

402 documented that bacterial pathogens can multiply during epiphytic and apoplastic colonization,

403 even in nonhost plants (Ishiga et al., 2011; Rojas et al., 2012; Senthil-Kumar and Mysore, 2012).

404 Consistent with previous reports, the endophytic bacterial multiplication was detected in the

405 Arabidopsis Col-0 wild-type inoculated with nonhost pathogens (Figs. 1A, 1B, and 1C),

406 suggesting that these bacterial pathogens may have potential for multiplication to a certain level

407 in the apoplastic space of nonhost plants. Therefore, it is tempting to speculate that the nutrients

408 derived from tissues showing enhanced LCD enable $P$. syringae pathovars to multiply during the

409 necrotrophic growth phase.

410 Our expression profiles and phytohormone analyses revealed the activation of genes related to

411 the JA biosynthesis pathway, including $L O X 2$ and $O P R 3$ (Figs. 4C and 4D), and the concomitant

412 accumulation of JA and JA-Ile in the ntrc mutant in response to a nonhost bacterial pathogen

413 (Figs. 3B and 3C). JA-mediated signaling pathways have been well characterized because of

414 their importance in plant defense systems in biotic and abiotic stress response. JA is known to

415 activate the plant defense system in response to wounding, herbivory, attack by necrotrophic

416 pathogens, and environmental stresses including low temperature, salinity, and drought (Campos

417 et al., 2014; Savatin et al., 2014; Riemann et al., 2015; Sharma and Laxmi, 2015). Consistent

418 with our results, Demmig-Adams et al. (2013) showed that Arabidopsis knockout mutants of key

419 components of the chloroplast photoprotection system produced high levels of JA and its

420 precursor 12-oxo phytodienoic acid (OPDA) (Demmig-Adams et al., 2013). Moreover, it has

421 been reported that the expression of genes related to the JA-mediated signaling pathway were

422 induced in response to high light stress in Arabidopsis (Tikkanen et al., 2014). It is well-known

423 that abiotic stresses including high light induce the generation of ROS in the chloroplast (Suzuki 
424 et al., 2012). Therefore, the chloroplast is considered to contain a large amount of

425 polyunsaturated fatty acids, the precursors for JA and OPDA, which show sensitivity to

426 oxidation by ROS (Schaller and Stintzi, 2009). Together, our results suggest the correlation

427 between chloroplast-derived ROS and the JA-signaling pathway in the ntrc mutant in response to 428 a nonhost bacterial pathogen.

429 We also demonstrated the induction of genes related to key regulators for the JA-mediated 430 signaling pathway, including MYC2, GRX480, ANAC019, and JAZ3 in the ntrc mutant in 431 response to a nonhost bacterial pathogen (Figs. 4E, 4F, 4G, and 4H), indicating that the loss-of-

432 function of $N T R C$ resulted in the activation of the JA-mediated signaling pathway during nonhost disease resistance. It has been well studied that cross-talk between SA and JA plays an 434 important role in fine tuning plant defenses to enhance plant fitness, and that MYC2 functions as 435 a key regulator of cross-talk between SA and JA (Laurie-Berry et al., 2006; Pieterse et al., 2009; 436 Thaler et al., 2012). Zheng et al. (2012) reported that MYC2 bound to the promoter of the NAC 437 transcription factor $A N A C 019$ and activated its expression. Furthermore, ANAC019 was shown to suppress the expression of ICS (Zheng et al., 2012). Consistent with these reports, the expression profiles of SA-mediated signaling revealed lower levels of the ICSI and PRI transcripts in the ntrc mutant compared to the wild-type Col-0 during nonhost resistance (Figs.

$4414 \mathrm{~A}$ and 4B). In addition, our previous studies showed that the SA-mediated signaling pathway 442 played an important role in nonhost disease resistance against bacterial pathogens (Ishiga et al.,

443 2011). However, our phytohormone analyses showed that there were no significant differences in 444 SA accumulation between the wild-type Col-0 and the $n t r c$ mutant (Fig. 5A). Therefore, it is 445 possible that chloroplast-derived ROS may function as a signaling compound to regulate the JA446 pathway, but not the SA-pathway. 


\section{Conclusions}

449 This study characterized the function of NTRC with respect to the nonhost disease resistance of Arabidopsis against bacterial pathogens, and provides additional insights into the mechanism of plant innate immunity, especially the correlation between chloroplast-derived ROS and LCD. fully understand the complex mechanisms of nonhost disease resistance with reference to NTRC.

We thank Dr. Christina Baker for editing the manuscript.

\section{References}

459

460

461

462

463

464

465

466

467

468

469

470

471

472

473

474

475

476

477

478

479
Almeida NF, Yan S, Lindeberg M, Studholme DJ, Schneider DJ, Condon B, Liu H, Viana CJ, Warren A, Evans C, Kemen E, Maclean D, Angot A, Martin GB, Jones JD, Collmer A, Setubal JC, Vinatzer BA (2009) A draft genome sequence of Pseudomonas syringae pv. tomato T1 reveals a type III effector repertoire significantly divergent from that of Pseudomonas syringae pv. tomato DC3000. Molecular plant-microbe interactions : MPMI 22: 52-62

Bender CL, Alarcón-Chaidez F, Gross DC (1999) Pseudomonas syringae phytotoxins: mode of action, regulation, and biosynthesis by peptide and polyketide synthetases. Microbiol Mol Biol Rev 63: 266-292

Bernal-Bayard P, Hervás M, Cejudo FJ, Navarro JA (2012) Electron transfer pathways and dynamics of chloroplast NADPH-dependent thioredoxin reductase C (NTRC). J Biol Chem 287: 33865-33872

Bernal-Bayard P, Ojeda V, Hervás M, Cejudo FJ, Navarro JA, Velázquez-Campoy A, Pérez-Ruiz JM (2014) Molecular recognition in the interaction of chloroplast 2-Cys peroxiredoxin with NADPH-thioredoxin reductase C (NTRC) and thioredoxin $\mathrm{x}$. FEBS Lett 588: 4342-4347

Brunkard JO, Runkel AM, Zambryski PC (2015) Chloroplasts extend stromules independently and in response to internal redox signals. Proc Natl Acad Sci U S A 112: 10044-10049

Campos ML, Kang JH, Howe GA (2014) Jasmonate-triggered plant immunity. J Chem Ecol 40: 657-675

Caplan JL, Kumar AS, Park E, Padmanabhan MS, Hoban K, Modla S, Czymmek K, Dinesh-Kumar SP (2015) Chloroplast Stromules Function during Innate Immunity. Dev Cell 34: 45-57

Cejudo FJ, Ferrández J, Cano B, Puerto-Galán L, Guinea M (2012) The function of the NADPH thioredoxin reductase C-2-Cys peroxiredoxin system in plastid redox regulation and signalling. FEBS Lett 586: 29742980

Chi W, Feng P, Ma J, Zhang L (2015) Metabolites and chloroplast retrograde signaling. Curr Opin Plant Biol 25: 
480

481

482

483

484

485

486

487

488

489

490

491

492

493

494

495

496

497

498

499

500

501

502

503

504

505

506

507

508

509

510

511

512

513

514

515

516

517

518

519

520

521

522

523

524

525

526

527

528

529

530

531

532

533

534

535
$32-38$

Chi W, Sun X, Zhang L (2013) Intracellular signaling from plastid to nucleus. Annu Rev Plant Biol 64: 559-582

Coll NS, Epple P, Dangl JL (2011) Programmed cell death in the plant immune system. Cell Death Differ 18: 12471256

Cui H, Tsuda K, Parker JE (2014) Effector-Triggered Immunity: From Pathogen Perception to Robust Defense. Annu Rev Plant Biol

Demmig-Adams B, Cohu CM, Amiard V, Zadelhoff G, Veldink GA, Muller O, Adams WW (2013) Emerging trade-offs - impact of photoprotectants (PsbS, xanthophylls, and vitamin E) on oxylipins as regulators of development and defense. New Phytol 197: 720-729

Fan J, Doerner P (2012) Genetic and molecular basis of nonhost disease resistance: complex, yes; silver bullet, no. Curr Opin Plant Biol 15: 400-406

Gill SS, Tuteja N (2010) Reactive oxygen species and antioxidant machinery in abiotic stress tolerance in crop plants. Plant Physiol Biochem 48: 909-930

Gill US, Lee S, Mysore KS (2015) Host versus nonhost resistance: distinct wars with similar arsenals. Phytopathology 105: $580-587$

Greenberg JT, Yao N (2004) The role and regulation of programmed cell death in plant-pathogen interactions. Cell Microbiol 6: 201-211

Hok S, Attard A, Keller H (2010) Getting the most from the host: how pathogens force plants to cooperate in disease. Mol Plant Microbe Interact 23: 1253-1259

Hossain MA, Bhattacharjee S, Armin SM, Qian P, Xin W, Li HY, Burritt DJ, Fujita M, Tran LS (2015) Hydrogen peroxide priming modulates abiotic oxidative stress tolerance: insights from ROS detoxification and scavenging. Front Plant Sci 6: 420

Ishiga Y, Ishiga T, Uppalapati SR, Mysore KS (2011) Arabidopsis seedling flood-inoculation technique: a rapid and reliable assay for studying plant-bacterial interactions. Plant Methods 7: 32

Ishiga Y, Ishiga T, Uppalapati SR, Mysore KS (2013) Jasmonate ZIM-domain (JAZ) protein regulates host and nonhost pathogen-induced cell death in tomato and Nicotiana benthamiana. PLoS One 8: e75728

Ishiga Y, Ishiga T, Wangdi T, Mysore KS, Uppalapati SR (2012) NTRC and chloroplast-generated reactive oxygen species regulate Pseudomonas syringae pv. tomato disease development in tomato and arabidopsis. Mol Plant Microbe Interact 25: 294-306

Ishiga Y, Uppalapati SR, Ishiga T, Elavarthi S, Martin B, Bender CL (2009) The phytotoxin coronatine induces light-dependent reactive oxygen species in tomato seedlings. New Phytol 181: 147-160

Keane PJ, Kerr A, New PB (1970) Crown gall of stone fruit. II. Identification and nomenclature of Agrobacterium isolates. Australian Journal of Biological Sciences 23: 585-595

Kirchsteiger K, Pulido P, González M, Cejudo FJ (2009) NADPH Thioredoxin reductase C controls the redox status of chloroplast 2-Cys peroxiredoxins in Arabidopsis thaliana. Mol Plant 2: 298-307

Laurie-Berry N, Joardar V, Street IH, Kunkel BN (2006) The Arabidopsis thaliana JASMONATE INSENSITIVE 1 gene is required for suppression of salicylic acid-dependent defenses during infection by Pseudomonas syringae. Mol Plant Microbe Interact 19: 789-800

Lee S, Ishiga Y, Clermont K, Mysore KS (2013) Coronatine inhibits stomatal closure and delays hypersensitive response cell death induced by nonhost bacterial pathogens. PeerJ 1: e34

Lim CJ, Kim WB, Lee BS, Lee HY, Kwon TH, Park JM, Kwon SY (2010) Silencing of SlFTR-c, the catalytic subunit of ferredoxin:thioredoxin reductase, induces pathogenesis-related genes and pathogen resistance in tomato plants. Biochem Biophys Res Commun 399: 750-754

Liu Y, Ren D, Pike S, Pallardy S, Gassmann W, Zhang S (2007) Chloroplast-generated reactive oxygen species are involved in hypersensitive response-like cell death mediated by a mitogen-activated protein kinase cascade. Plant J 51: 941-954

Loehrer M, Langenbach C, Goellner K, Conrath U, Schaffrath U (2008) Characterization of nonhost resistance of Arabidopsis to the Asian soybean rust. Mol Plant Microbe Interact 21: 1421-1430

Mur LA, Kenton P, Lloyd AJ, Ougham H, Prats E (2008) The hypersensitive response; the centenary is upon us but how much do we know? J Exp Bot 59: 501-520

Mysore KS, Ryu CM (2004) Nonhost resistance: how much do we know? Trends Plant Sci 9: 97-104

Newton AC, Fitt BD, Atkins SD, Walters DR, Daniell TJ (2010) Pathogenesis, parasitism and mutualism in the trophic space of microbe-plant interactions. Trends Microbiol 18: 365-373

Niks RE, Marcel TC (2009) Nonhost and basal resistance: how to explain specificity? New Phytol 182: 817-828

Nomura H, Komori T, Uemura S, Kanda Y, Shimotani K, Nakai K, Furuichi T, Takebayashi K, Sugimoto T, Sano S, Suwastika IN, Fukusaki E, Yoshioka H, Nakahira Y, Shiina T (2012) Chloroplast-mediated 
activation of plant immune signalling in Arabidopsis. Nat Commun 3: 926

Oh SK, Lee S, Chung E, Park JM, Yu SH, Ryu CM, Choi D (2006) Insight into Types I and II nonhost resistance using expression patterns of defense-related genes in tobacco. Planta 223: 1101-1107

Palmer DA, Bender CL (1995) Ultrastructure of tomato leaf tissue treated with the Pseudomonad phytotoxin coronatine and comparison with methyl jasmonate. In, Vol 8(5):683 Mol Plant Microbe Interact, p 683

Perez-Ruiz JM, Spinola MC, Kirchsteiger K, Moreno J, Sahrawy M, Cejudo FJ (2006) Rice NTRC is a highefficiency redox system for chloroplast protection against oxidative damage. Plant Cell 18: 2356-2368

Pieterse CM, Leon-Reyes A, Van der Ent S, Van Wees SC (2009) Networking by small-molecule hormones in plant immunity. Nat Chem Biol 5: 308-316

Pieterse CM, Van der Does D, Zamioudis C, Leon-Reyes A, Van Wees SC (2012) Hormonal modulation of plant immunity. Annu Rev Cell Dev Biol 28: 489-521

Puerto-Galán L, Pérez-Ruiz JM, Guinea M, Cejudo FJ (2015) The contribution of NADPH thioredoxin reductase C (NTRC) and sulfiredoxin to 2-Cys peroxiredoxin overoxidation in Arabidopsis thaliana chloroplasts. J Exp Bot 66: 2957-2966

Riemann M, Dhakarey R, Hazman M, Miro B, Kohli A, Nick P (2015) Exploring Jasmonates in the Hormonal Network of Drought and Salinity Responses. Front Plant Sci 6: 1077

Robert-Seilaniantz A, Grant M, Jones JD (2011) Hormone crosstalk in plant disease and defense: more than just jasmonate-salicylate antagonism. Annu Rev Phytopathol 49: 317-343

Rojas CM, Senthil-Kumar M, Wang K, Ryu CM, Kaundal A, Mysore KS (2012) Glycolate oxidase modulates reactive oxygen species-mediated signal transduction during nonhost resistance in Nicotiana benthamiana and Arabidopsis. Plant Cell 24: 336-352

Rolland N, Curien G, Finazzi G, Kuntz M, Maréchal E, Matringe M, Ravanel S, Seigneurin-Berny D (2012) The biosynthetic capacities of the plastids and integration between cytoplasmic and chloroplast processes. Annu Rev Genet 46: 233-264

Savatin DV, Gramegna G, Modesti V, Cervone F (2014) Wounding in the plant tissue: the defense of a dangerous passage. Front Plant Sci 5: 470

Schaller A, Stintzi A (2009) Enzymes in jasmonate biosynthesis - structure, function, regulation. Phytochemistry 70: $1532-1538$

Senthil-Kumar M, Mysore KS (2012) Ornithine-delta-aminotransferase and proline dehydrogenase genes play a role in non-host disease resistance by regulating pyrroline-5-carboxylate metabolism-induced hypersensitive response. Plant Cell Environ 35: 1329-1343

Senthil-Kumar M, Mysore KS (2013) Nonhost resistance against bacterial pathogens: retrospectives and prospects. Annu Rev Phytopathol 51: 407-427

Seyfferth C, Tsuda K (2014) Salicylic acid signal transduction: the initiation of biosynthesis, perception and transcriptional reprogramming. Front Plant Sci 5: 697

Sharma M, Laxmi A (2015) Jasmonates: Emerging Players in Controlling Temperature Stress Tolerance. Front Plant Sci 6: 1129

Staskawicz BJ, Dahlbeck D, Keen NT (1984) Cloned avirulence gene of Pseudomonas syringae pv. glycinea determines race-specific incompatibility on Glycine max (L.) Merr. Proceedings of the National Academy of Sciences of the United States of America 81: 6024-6028

Suzuki N, Koussevitzky S, Mittler R, Miller G (2012) ROS and redox signalling in the response of plants to abiotic stress. Plant Cell Environ 35: 259-270

Tada Y, Spoel SH, Pajerowska-Mukhtar K, Mou Z, Song J, Wang C, Zuo J, Dong X (2008) Plant immunity requires conformational changes [corrected] of NPR1 via S-nitrosylation and thioredoxins. Science 321: 952956

Taguchi F, Tanaka R, Kinoshita S, Ichinose Y, Imura Y, Andi S, Toyoda K, Shiraishi T, Yamada T (2001) HarpinPsta from Pseudomonas syringae pv. tabaci is defective and deficient in its expression and HRinducing activity. Journal of general plant pathology: 116-123

Thaler JS, Humphrey PT, Whiteman NK (2012) Evolution of jasmonate and salicylate signal crosstalk. Trends Plant Sci 17: 260-270

Tikkanen M, Gollan PJ, Mekala NR, Isojärvi J, Aro EM (2014) Light-harvesting mutants show differential gene expression upon shift to high light as a consequence of photosynthetic redox and reactive oxygen species metabolism. Philos Trans R Soc Lond B Biol Sci 369: 20130229

Tripathi BN, Bhatt I, Dietz KJ (2009) Peroxiredoxins: a less studied component of hydrogen peroxide detoxification in photosynthetic organisms. Protoplasma 235: 3-15

Tsukahara K, Sawada H, Kohno Y, Matsuura T, Mori IC, Terao T, Ioki M, Tamaoki M (2015) Ozone-Induced 
592

593

594

595

596

597

598

599

600

601

602

603

604

605

606

607

608

609

610

611

612

613

614

615

616

617

618

619

620

621

622

Rice Grain Yield Loss Is Triggered via a Change in Panicle Morphology That Is Controlled by ABERRANT PANICLE ORGANIZATION 1 Gene. PLoS One 10: e0123308

Wasternack C, Hause B (2013) Jasmonates: biosynthesis, perception, signal transduction and action in plant stress response, growth and development. An update to the 2007 review in Annals of Botany. Ann Bot 111: 10211058

Zheng XY, Spivey NW, Zeng W, Liu PP, Fu ZQ, Klessig DF, He SY, Dong X (2012) Coronatine promotes Pseudomonas syringae virulence in plants by activating a signaling cascade that inhibits salicylic acid accumulation. Cell Host Microbe 11: 587-596

Zipfel C (2008) Pattern-recognition receptors in plant innate immunity. Curr Opin Immunol 20: 10-16

Zipfel C, Felix G (2005) Plants and animals: a different taste for microbes? Curr Opin Plant Biol 8: 353-360

Zurbriggen MD, Carrillo N, Tognetti VB, Melzer M, Peisker M, Hause B, Hajirezaei MR (2009) Chloroplastgenerated reactive oxygen species play a major role in localized cell death during the non-host interaction between tobacco and Xanthomonas campestris pv. vesicatoria. Plant J 60: 962-973

Figure legends

\section{Figure 1: Analysis of the nonhost resistance responses of the Arabidopsis thaliana wild-type}

\section{Col-0, and the prx (prxa, prxb, prxq and pexIIe) and ntrc mutants using a seedling flood-}

inoculation assay. (A) Response of the Arabidopsis thaliana wild-type Col-0, and the prx (prxa,

prxb, prxq, and pexIIe) and ntrc mutants to nonhost Pseudomonas syringae pathogens.

Arabidopsis seedlings were flood-inoculated with nonhost pathogens including Pseudomonas

syringae pv. tabaci (Pta 6605), P. syringae pv. glycinea (Pgl race 4), and P. syringae pv. tomato

T1 (Pto T1) at a concentration of $5 \times 10^{6} \mathrm{CFU} / \mathrm{ml}$. Photographs were taken at $2 \mathrm{dpi}$. (B) Ion

leakage from the Arabidopsis thaliana wild-type Col-0, and the prx (prxa, prxb, prxq, and

pexIle) and ntrc mutants flooded with water (mock) or Pseudomonas syringae pv. tabaci (Pta

6605), P. syringae pv. glycinea (Pgl race 4), and P. syringae pv. tomato T1 (Pto T1) at a

concentration of $5 \times 10^{6} \mathrm{CFU} / \mathrm{ml}$. Samples were collected at 2 dpi. Bars show the percentage of

total ions. Vertical bars indicate the standard error for three biological replicates. Statistically

significant differences are noted as different alphabet characters based on ANOVA $(p<0.05)$.

Bacterial populations of (C) Pseudomonas syringae pv. tabaci (Pta 6605), (D) P. syringae pv.

glycinea (Pgl race 4), and (E) P. syringae pv. tomato T1 (Pto T1) in the Arabidopsis wild-type

Col-0, and the prx (prxa, prxb, prxq, and pexIIe) and ntrc mutants. Bacterial populations were

quantified at 0 and 2 dpi. Vertical bars indicate the standard errors for three independent 
623 experiments. Asterisks indicate a significant difference from the wild-type Col-0 in a $t$-test $(*=p$

$624<0.05, * *=p<0.01)$

625 Figure 2: Hydrogen peroxide production in the Arabidopsis wild-type Col-0, prx (prxa, 626 prxb, prxq, and pexIIe) and ntrc mutants in response to Pseudomonas syringae pv. tabaci

627 (Pta 6605), P. syringae pv. glycinea (Pgl race 4), and P. syringae pv. tomato T1 (Pto T1) at a

628 concentration of $5 \times \mathbf{1 0}^{6} \mathbf{C F U} / \mathbf{m l}$. (A) Hydrogen peroxide production in Arabidopsis

629 chloroplast-localized prx and ntrc mutants in response to nonhost $P$. syringae pathogens. ROS

630 were visualized by staining hydrogen peroxide using 3,3'-diaminobenzidine at 2 dpi. (B) The

631 intensity of DAB staining shown in panel $\mathrm{A}$ is quantified and expressed as a percentage of

632 coloration, where the color intensity of mock-treated wild-type Col-0 leaves was set at 100\%.

633 Vertical bars indicate the standard error for 10 leaves. Statistically significant differences are

634 noted as different alphabet characters based on ANOVA $(p<0.05)$.

635 Figure 3: Quantification of phytohormones including salicylic acid (SA; A), jasmonic acid

636 (JA; B), and jasmonoyl-L-isoleucine (JA-Ile; C) in the Arabidopsis wild-type (Col-0), ntrc

637 mutant, and its complemented line (35S-NTRC/ntrc). Two-week-old Col-0, ntrc, and 35S-

638 NTRC/ntrc were treated with water as a mock control (Mock) or inoculated with nonhost

639 pathogen Pseudomonas syringae pv. tabaci (Pta 6605) at a concentration of $5 \times 10^{6} \mathrm{CFU} / \mathrm{ml}$ for

$64024 \mathrm{~h}$, and phytohormone quantification was performed. Vertical bars indicate the standard errors

641 for four biological replicates. Asterisks indicate a significant difference from the wild-type Col-0

642 in a $t$-test $(*=p<0.05)$.

643 Figure 4: Expression profiles of genes encoding for the SA- and JA-mediated signaling

644 pathways in the Arabidopsis wild-type (Col-0), ntrc mutant, and its complement line (35S-

645 NTRC/ntrc). The expression profiles of the SA-pathway genes including ISOCHORISMATE 
646 SYNTHASE 1 (A) and PATHOGENESIS-RELATED PROTEIN 1 (B), and JA-pathway genes

647 including LIPOXYGENASE 2 (C), 12-OXOPHYTODIENOIC ACID REDUCTASE 3 (D), MYC2

648 (E), GLUTAREDOXIN PROTEIN 480 (F), NAC DOMAIN CONTAINING PROTEIN 19 (G), and 649 JASMONATE-ZIM-DOMAIN PROTEIN 3 (H) were obtained at 3, 6, 12, and $24 \mathrm{~h}$ after

650 inoculation with the nonhost pathogen Pseudomonas syringae pv. tabaci (Pta 6605). Two-week651 old Arabidopsis Col-0, ntrc, and 35S-NTRC/ntrc plants were treated with water as a mock 652 control (Mock), or inoculated with nonhost Pta 6605at a concentration of $5 \times 10^{6} \mathrm{CFU} / \mathrm{ml}$. The 653 expression of genes was evaluated by RT-qPCR with gene-specific primer sets (Supplementary 654 Table S1). The values represent the relative induction compared to the expression of $U B Q 1$. 655 Figures are from a representative experiment and repeated at least 3 times with similar results. 656 Vertical bars indicate the standard error for three biological replicates. Statistically significant 657 differences are noted as different alphabet characters based on ANOVA $(p<0.05)$.

658 Supplementary Figure S1: Analysis of the nonhost resistance responses of the Arabidopsis 659 thaliana wild-type Col-0, ntrc mutant, and its complemented line (35S-NTRC/ntrc) using a 660 seedling flood-inoculation assay. (A) Response of the Arabidopsis thaliana wild-type Col-0, 661 ntrc mutant, and the complemented line (35S-NTRC/ntrc) to nonhost Pseudomonas syringae 662 pathogens. Arabidopsis seedlings were individually flood-inoculated with nonhost pathogens 663 including Pseudomonas syringae pv. tabaci (Pta 6605), P. syringae pv. glycinea (Pgl race 4), 664 and P. syringae pv. tomato T1 (Pto T1) at a concentration of $5 \times 10^{6} \mathrm{CFU} / \mathrm{ml}$. Photographs were 665 taken at 2 dpi. (B) Ion leakage from the Arabidopsis thaliana wild-type Col-0, ntrc mutant, and 666 the complemented line (35S-NTRC/ntrc) flooded with water (mock), or Pseudomonas syringae 667 pv. tabaci (Pta 6605), P. syringae pv. glycinea (Pgl race 4), or P. syringae pv. tomato T1 (Pto $668 \mathrm{~T} 1)$ at a concentration of $5 \times 10^{6} \mathrm{CFU} / \mathrm{ml}$. Samples were collected at $2 \mathrm{dpi}$. Bars show the 
669 percentage of total ions. Vertical bars indicate the standard error for three biological replicates.

670 Statistically significant differences are noted as different alphabet characters based on ANOVA

$671(p<0.05)$. (C) Bacterial populations of Pseudomonas syringae pv. tabaci (Pta 6605), P.

672 syringae pv. glycinea (Pgl race 4), and P. syringae pv. tomato T1 (Pto T1) in the Arabidopsis

673 wild-type Col-0, ntrc mutant, and the complement line (35S-NTRC/ntrc). Bacterial populations

674 were quantified at 0 and 2 dpi. Vertical bars indicate the standard errors for three independent

675 experiments. Asterisks indicate a significant difference from the wild-type Col-0 in a $t$-test $(*=p$

$676<0.05, * *=p<0.01$ ). (D) Hydrogen peroxide production in the Arabidopsis wild-type Col-0,

677 ntrc mutant, and the complemented line (35S-NTRC/ntrc) in response to $P$. syringae pv. tabaci

678 (Pta 6605), P. syringae pv. glycinea (Pgl race 4), and P. syringae pv. tomato T1 (Pto T1) at a

679 concentration of $5 \times 10^{6} \mathrm{CFU} / \mathrm{ml}$. ROS were visualized by staining hydrogen peroxide using

680 3,3'-diaminobenzidine at 2 dpi. (E) The intensity of DAB staining shown in panel D is

681 quantified and expressed as a percentage of coloration, where the color intensity of mock-treated

682 wild-type Col-0 leaves was set at 100\%. Vertical bars indicate the standard error for 10 leaves.

683 Statistically significant differences are noted as different alphabet characters based on ANOVA

$684(p<0.05)$.

685 Supplementary Figure S2: Hydrogen peroxide production in the Arabidopsis wild-type Col-

686 0, ntrc mutant, and its complemented line (35S-NTRC/ntrc) in response to Pseudomonas

687 syringae pv. tabaci (Pta 6605), P. syringae pv. glycinea (Pgl race 4), and P. syringae pv.

688 tomato T1 (Pto T1) at a concentration of $5 \times 10^{6}$ CFU/ml. ROS were visualized by staining

689 hydrogen peroxide using 3,3'-diaminobenzidine at 1 dpi. The intensity of DAB staining is

690 quantified and expressed as a percentage of coloration, where the color intensity of mock-treated

691 wild-type Col-0 leaves was set at 100\%. Vertical bars indicate the standard error for 10 leaves. 
692 Statistically significant differences are noted as different alphabet characters based on ANOVA $693(p<0.05)$.

694 Supplementary Figure S3: Expression profiles of genes encoding for the chloroplast695 localized ROS detoxifying enzymes in the Arabidopsis wild-type (Col-0), ntrc mutant, and 696 its complemented line (35S-NTRC/ntrc). The expression profiles of the genes related to ROS

697 detoxification including STROMAL ASCORBATE PEROXIDASE (A), THYLAKOIDAL 698 ASCORBATE PEROXIDASE (B), GLUTATHIONE PEROXIDASE 1 (C), and GLUTATHIONE 699 PEROXIDASE 7 (D) were obtained at 3, 6, 12, and $24 \mathrm{~h}$ after inoculation with the nonhost 700 pathogen Pseudomonas syringae pv. tabaci (Pta 6605). Two-week-old Arabidopsis Col-0, ntrc, 701 and 35S-NTRC/ntrc plants were treated with water as a mock control (Mock), or inoculated with 702 Pta 6605 at a concentration of $5 \times 10^{6} \mathrm{CFU} / \mathrm{ml}$. The expression of genes was evaluated by RT703 qPCR with gene-specific primer sets (Supplementary Table S1). The values represent the relative 704 induction compared to the expression of $U B Q 1$. The figures are from a representative experiment 705 and were repeated at least 3 times with similar results. Vertical bars indicate the standard error

706 for three biological replicates. Statistically significant differences are noted as different alphabet 707 characters based on ANOVA $(p<0.05)$. 


\section{Figure 1 (on next page)}

Analysis of the nonhost resistance responses of the Arabidopsis thaliana wild-type Col-0, and the prx (prxa, prxb, prxq and pexlle) and ntrc mutants using a seedling floodinoculation assay.

(A) Response of the Arabidopsis thaliana wild-type Col-0, and the prx (prxa, prxb, prxq, and pexlle) and ntrc mutants to nonhost Pseudomonas syringae pathogens. Arabidopsis seedlings were flood-inoculated with nonhost pathogens including Pseudomonas syringae pv. tabaci ( $P$. s. pv. tabaci), P. syringae pv. glycinea (P. s. pv. glycinea), and P. syringae pv. tomato T1 (P. s. pv. tomato T1) at a concentration of $5 \times 10^{6} \mathrm{CFU} / \mathrm{ml}$. Photographs were taken at $2 \mathrm{dpi}$. (B) Ion leakage from the Arabidopsis thaliana wild-type Col-0, and the prx (prxa, prxb, prxq, and pexlle) and ntrc mutants flooded with water (mock) or Pseudomonas syringae pv. tabaci ( $P$. s. pv. tabaci), P. syringae pv. glycinea (P. s. pv. glycinea), and P. syringae pv. tomato T1 (P. s. pv. tomato T1) at a concentration of $5 \times 10^{6} \mathrm{CFU} / \mathrm{ml}$. Samples were collected at $2 \mathrm{dpi}$. Bars show the percentage of total ions. Vertical bars indicate the standard error for three biological replicates. Statistically significant differences are noted as different alphabet characters based on ANOVA $(p<0.05)$. Bacterial populations of (C) Pseudomonas syringae pv. tabaci (P. s. pv. tabaci), (D) P. syringae pv. glycinea (P. s. pv. glycinea), and (E) P. syringae pv. tomato T1 (P. s. pv. tomato T1) in the Arabidopsis wild-type Col-0, and the prx (prxa, prxb, prxq, and pexlle) and ntrc mutants. Bacterial populations were quantified at 0 and $2 \mathrm{dpi}$. Vertical bars indicate the standard errors for three independent experiments. Asterisks indicate a significant difference from the wild-type Col-0 in a t-test $*^{*}=p<0.05,{ }^{*}$ $=p<0.01$. . 


\section{Figure 2 (on next page)}

Hydrogen peroxide production in the Arabidopsis wild-type Col-0, prx (prxa, prxb, prxq, and pexlle) and ntrc mutants in response to Pseudomonas syringae pathogens.

(A) Hydrogen peroxide production in Arabidopsis chloroplast-localized prx and ntrc mutants in response to nonhost $P$. syringae pathogens. ROS were visualized by staining hydrogen peroxide using 3,3'-diaminobenzidine at $2 \mathrm{dpi}$. (B) The intensity of DAB staining shown in panel $\mathrm{A}$ is quantified and expressed as a percentage of coloration, where the color intensity of mock-treated wild-type Col-0 leaves was set at $100 \%$. Vertical bars indicate the standard error for 10 leaves. Statistically significant differences are noted as different alphabet characters based on ANOVA $(p<0.05)$. 
PeerJ

Mock Pta 6605 Pgl race 4 Pto T1

Col-0

prxa

prxb
Manuscript to be reviewed

Mock Pta 6605 Pgl race 4 Pto T1

prxq

prxlle

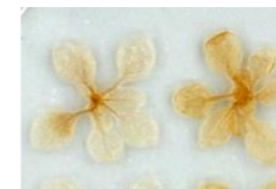

af

ntrc

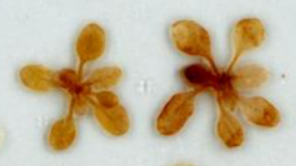

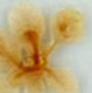

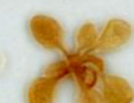

(B)

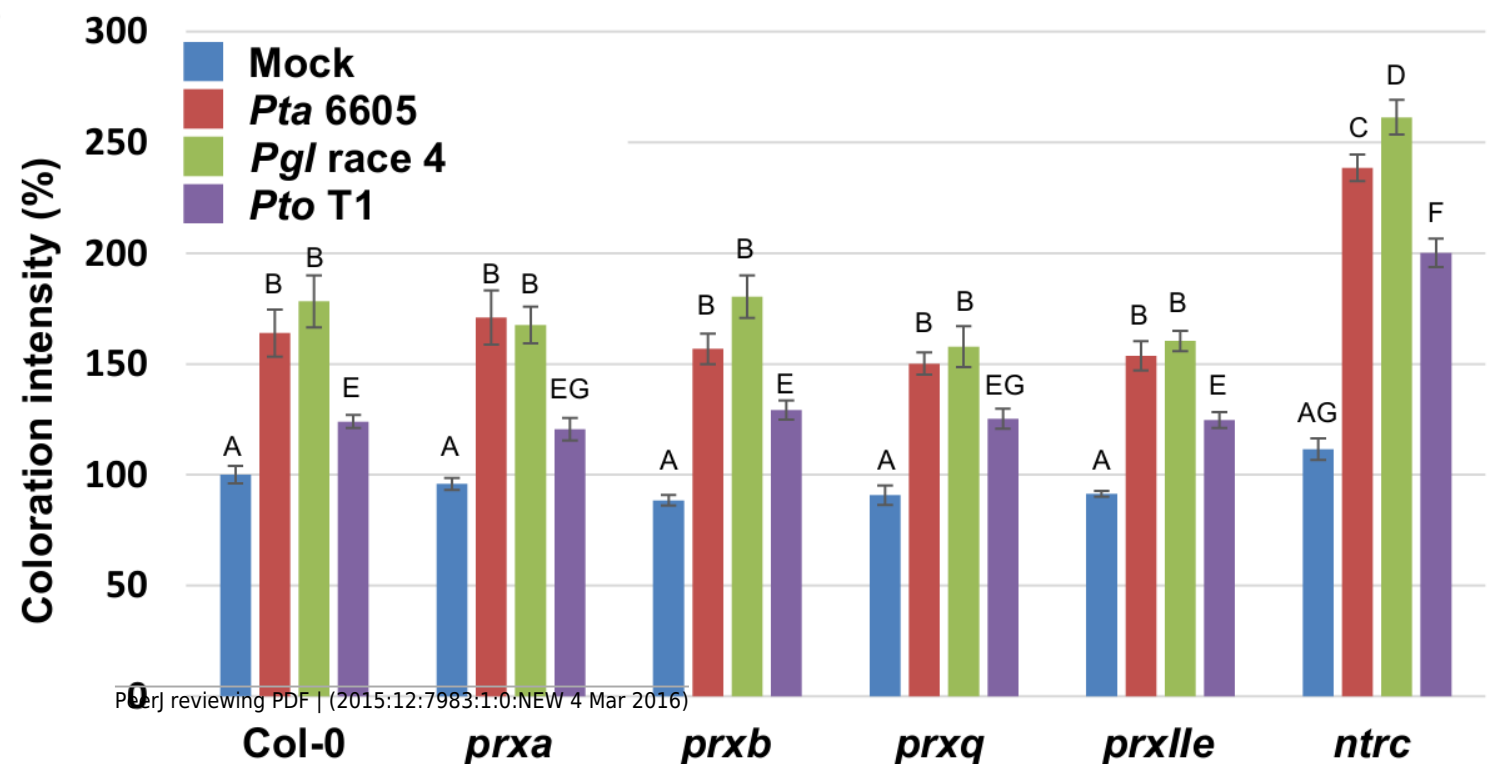




\section{Figure 3 (on next page)}

Quantification of phytohormones including salicylic acid (SA; A), jasmonic acid (JA; B), and jasmonoyl-L-isoleucine (JA-Ile; $\mathrm{C}$ ) in the Arabidopsis wild-type (Col-0), ntrc mutant, and its complemented line (35S-NTRC/ntrC).

Two-week-old Col-0, ntrc, and 35S-NTRC/ntrc were treated with water as a mock control (Mock) or inoculated with nonhost pathogen Pseudomonas syringae pv. tabaci (P. s. tabaci) at a concentration of $5 \times 10^{6} \mathrm{CFU} / \mathrm{ml}$ for $24 \mathrm{~h}$, and phytohormone quantification was performed. Vertical bars indicate the standard errors for four biological replicates. Asterisks indicate a significant difference from the wild-type Col-0 in a t-test $(*=p<0.05)$. 

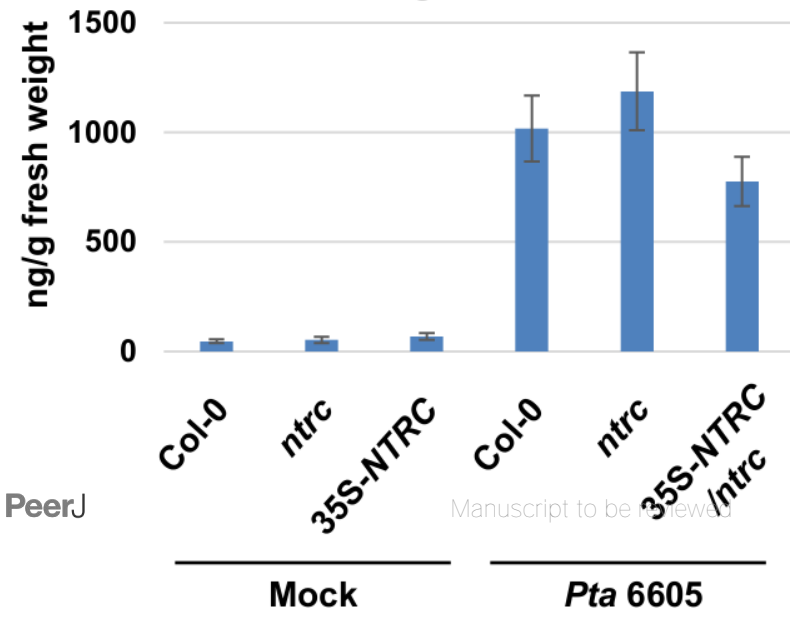

(B)

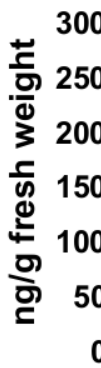

JA

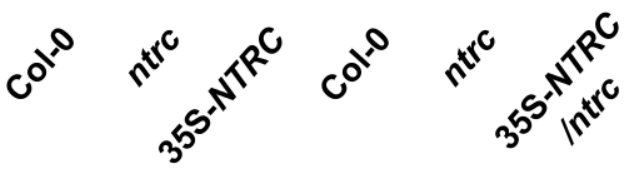

Mock

Pta 6605

(C)

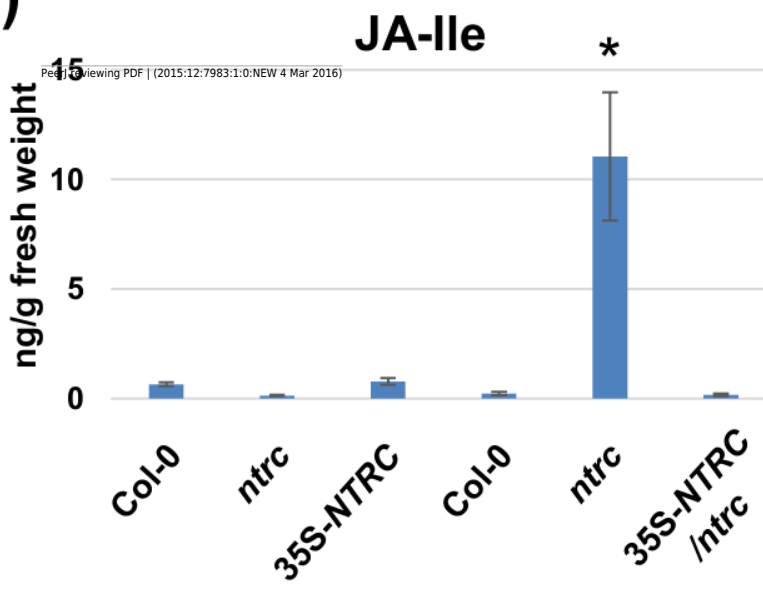




\section{Figure 4 (on next page)}

Expression profiles of genes encoding for the SA- and JA-mediated signaling pathways in the Arabidopsis wild-type (Col-0), ntrc mutant, and its complement line (35S-NTRC/ntrc).

The expression profiles of the SA-pathway genes including ISOCHORISMATE SYNTHASE 1 (A) and PATHOGENESIS-RELATED PROTEIN 1 (B), and JA-pathway genes including LIPOXYGENASE 2 (C), 12-OXOPHYTODIENOIC ACID REDUCTASE 3 (D), MYC2 (E), GLUTAREDOXIN PROTEIN 480 (F), NAC DOMAIN CONTAINING PROTEIN 19 (G), and JASMONATE-ZIM-DOMAIN PROTEIN 3 (H) were obtained at $3,6,12$, and $24 \mathrm{~h}$ after inoculation with the nonhost pathogen Pseudomonas syringae pv. tabaci. Two-week-old Arabidopsis Col-0, ntrc, and 35S-NTRC/ntrc plants were treated with water as a mock control (Mock), or inoculated with nonhost $P$. syringae pv. tabaci at a concentration of $5 \times 10^{6} \mathrm{CFU} / \mathrm{ml}$. The expression of genes was evaluated by RT-qPCR with gene-specific primer sets (Supplementary Table S1). The values represent the relative induction compared to the expression of $U B Q 1$. Figures are from a representative experiment and repeated at least 3 times with similar results. Vertical bars indicate the standard error for three biological replicates. Statistically significant differences are noted as different alphabet characters based on ANOVA $(p<0.05)$. 
